\title{
Our Valuable Contributors: Reviewers of 2015
}

I would like to recognize the peer reviewers who donated their precious time and effort to reviewing the manuscripts submitted to the Journal of Preventive Medicine and Public Health in 2015. With an ever increasing competition to attract quality research by major journals in our field, we depend on the expertise of the reviewers to critically appraise the papers. Their comments, detailed and extensive, guide authors to polish their work and propel them to strive for excellence. Their commitment and dedication to this hard and time consuming task ensure that published research are relevant, timely, high quality, and applicable.

I would like to extend my sincere gratitude to each and every one of the 64 reviewers.

Sung-il Cho, MD, ScD

Editor-in-Chief

$\begin{array}{lll}\text { Franz Allerberger } & \text { Chang-yup Kim } & \text { Hyun-Sul Lim } \\ \text { Hae-Kwan Cheong } & \text { Dong-Hyun Kim } & \text { Hyunwoo Lim } \\ \text { Kyungho Choi } & \text { Heon Kim } & \text { Jungsu Lim } \\ \text { Yong Jun Choi } & \text { Ho Kim } & \text { Baeg-Ju Na } \\ \text { Jin-Ho Chun } & \text { Hyeon Chang Kim } & \text { Chung Mo Nam } \\ \text { Young Kyung Do } & \text { Hyung Su Kim } & \text { Hae-Sung Nam } \\ \text { Sang Jun Eun } & \text { Jang-Rak Kim } & \text { In-Hwan Oh } \\ \text { Mina Ha } & \text { Jong Yeon Kim } & \text { Byung-Joo Park } \\ \text { Dongwoon Han } & \text { Kyoo Sang Kim } & \text { Jong Hyock Park } \\ \text { Jee Young Hong } & \text { Myoung-Hee Kim } & \text { Ki Soo Park } \\ \text { Young-Seoub Hong } & \text { Tae-Hyun Kim } & \text { Soon-Woo Park } \\ \text { Seung Sik Hwang } & \text { Yong-Dae Kim } & \text { Aesun Shin } \\ \text { Sun Ha Jee } & \text { Yoon Kim } & \text { Hee-Young Shin } \\ \text { Gyeong-Suk Jeon } & \text { Kwang-Pil Ko } & \text { Hosung Shin } \\ \text { Hyoung-Sun Jeong } & \text { Kwang-Wook Koh } & \text { Jeong Woo Sonn } \\ \text { Min-Woo Jo } & \text { Keun Sang Kwon } & \text { Jae Woong Sull } \\ \text { Shin Kam } & \text { Sun Seog Kwon } & \text { Jong Min Woo } \\ \text { Yun Sik Kang } & \text { Duk-Hee Lee } & \text { Seunghyun Yoo } \\ \text { Young Ho Khang } & \text { Kun Sei Lee } & \text { Taiyoung Yoon } \\ \text { Moran Ki } & \text { Moo-Sik Lee } & \text { Yongwoon Yun } \\ \text { Changhoon Kim } & \text { Sang Gyu Lee } & \\ \text { Changsu Kim } & \text { Soon-Young Lee } & \\ & & \end{array}$

